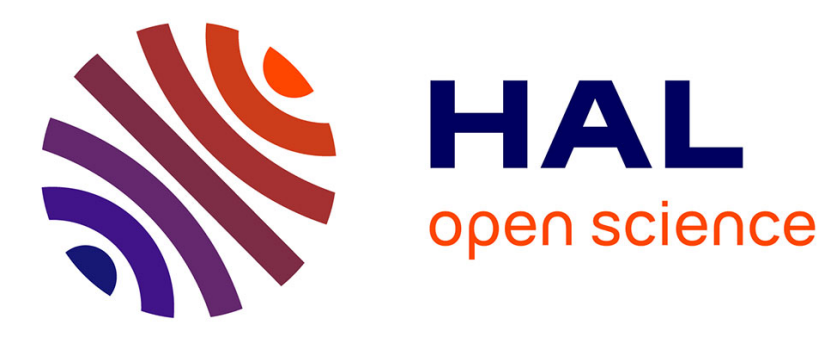

\title{
Flattening Earth acceleration in atomic fountains
}

Andrea Bertoldi

\section{To cite this version:}

Andrea Bertoldi. Flattening Earth acceleration in atomic fountains. Physical Review A: Atomic, molecular, and optical physics [1990-2015], 2010, 82, pp.013622. 10.1103/PhysRevA.82.013622 . hal00628521

\section{HAL Id: hal-00628521 \\ https://hal-iogs.archives-ouvertes.fr/hal-00628521}

Submitted on 4 Dec 2015

HAL is a multi-disciplinary open access archive for the deposit and dissemination of scientific research documents, whether they are published or not. The documents may come from teaching and research institutions in France or abroad, or from public or private research centers.
L'archive ouverte pluridisciplinaire HAL, est destinée au dépôt et à la diffusion de documents scientifiques de niveau recherche, publiés ou non, émanant des établissements d'enseignement et de recherche français ou étrangers, des laboratoires publics ou privés. 


\title{
Flattening Earth acceleration in atomic fountains
}

\author{
Andrea Bertoldi \\ Laboratoire Charles Fabry de l'Institut d'Optique, Univ. Paris Sud, Campus Polytechnique RD128, F-91127 Palaiseau, France
}

(Received 5 February 2010; published 16 July 2010)

\begin{abstract}
A method to compensate for Earth's gravity tide over an extended axial region is reported. Flattening acceleration is important in experiments where the coupling of the dynamics of free-falling probes to the gravity gradient generates stochastic noise on the measurement. Optimized cylindrically symmetric mass distributions lower Earth's tidal effect over $10 \mathrm{~cm}$ by a factor $10^{3}$. A multimass compensation system with comparable performance is devised for tall atom interferometers. Reducing the gravity gradient is essential in terrestrial experiments based on atom fountain configurations being developed to precisely test general relativity or the neutrality of matter.
\end{abstract}

DOI: 10.1103/PhysRevA.82.013622

PACS number(s): 37.25.+k, 91.10.Qm, 98.35.Ce

\section{INTRODUCTION}

Cold atom fountains are nowadays important scientific and technological instruments, competing with the state of the art in several measurement fields. The cesium fountain clock [1] defines the standard time, and fountain-based inertial sensors [2,3] have achieved sensitivities and accuracies that compare favorably with competing methods. Such performances have been used to realize atom interferometry tests of general relativity [4,5], and measure fundamental constants and their time variation [6-8]. Other fountain-based experiments designed to carry out tests of the equivalence principle [9], measure matter neutrality [10], and detect gravitational waves [11] are presently under construction.

These experiments require precise knowledge of the trajectory of the free-falling atomic probe in Earth's gravitational field. The noise on the initial position and velocity of the probe translates into stochastic noise of the output measurement whenever it depends on the gravity acceleration $g(z)$; this happens because of the vertical inhomogeneity of Earth's gravitational field, which is expressed in terms of a vertical gravity gradient $\Gamma_{V}$. By solving the equation of motion in the gravitational potential $\phi(z)=g_{0} z+1 / 2 \Gamma_{V} z^{2}$, it turns out that a vertical displacement $\delta z$ of the probe at the beginning of the experiment has the same effect of changing the acceleration by $\delta g=\delta z \Gamma_{V}$ during all the free fall. On the other hand, an initial change in the vertical velocity $\delta v_{z}$ provides the effect analogous to changing the acceleration by $\delta g=\delta v_{z} \Gamma_{V} t$ at each time $t$ during the experimental sequence. The fluctuating initial position and velocity of the probe are a source of stochastic noise, which must be considered in the evaluation of the experimental sensitivity; its effect can be reduced by compensating the gravity gradient in the measurement region.

To date, the only fountain-based atom interferometer in which the uncertainty given by the atomic position in the gravitational field is reduced by using nearby compensation masses is the gravity gradiometer [7,12]. In this case, two interferometers are realized in the regions where the total vertical acceleration presents stationary points resulting from heavy source masses (whose main purpose is to provide the gravitational signal to measure $G$ ); in this way, the uncertainty on the phase measurement due to the atom positioning is scaled down by 2 orders of magnitude.
In general, producing a stationary point of the vertical acceleration solves the effect of the gravity gradient in experiments in which the probe is at a fixed height in Earth's gravitational field. In this case, an approach that aims to cancel out the gradient and successive terms in the Taylor expansion of the gravitational potential at that point can be adopted (e.g., [13] for Earth-based experiments, and [14,15] for free-fall orbiting ones).

In this article the case of a probe moving vertically during the experiment is considered, which turns the problem from flattening Earth's gravitational field at just one point to flattening it over an extended axial region. Given a set of physical and geometrical constraints, the best configuration of cylindrically symmetric masses that achieve this goal is sought. The sensitivity improvement due to the flattening of the acceleration is analyzed for three-pulse Raman atom interferometry, and the method is further adapted to reduce the phase noise generated by the gravity gradient in tall fountain-based atom interferometers.

\section{EARTH GRAVITY GRADIENT}

Earth's gravity gradient can be written as a second-order tensor. The main components of this tensor are diagonal when a coordinate system with the local vertical as one axis is adopted:

$$
\Gamma_{i j} \simeq\left(\begin{array}{ccc}
\Gamma_{H} & 0 & 0 \\
0 & \Gamma_{H} & 0 \\
0 & 0 & \Gamma_{V}
\end{array}\right),
$$

where $\Gamma_{H}$ and $\Gamma_{V}$ are a compression orthogonal to the vertical axis and a tension along it, given by

$$
\begin{gathered}
\Gamma_{H}=-\frac{G M}{(R+h)^{3}}, \\
\Gamma_{V}=\frac{2 G M}{(R+h)^{3}} .
\end{gathered}
$$

In the previous equations, $M$ is the mass of Earth, $R$ its radius, $h$ the orbital altitude, and $G$ the Newtonian gravitational constant. Since Earth is not spherically symmetric, there are small variations in these principal components, and offdiagonal terms are not perfectly vanishing. In this article Earth is assumed to be perfectly spherical, and the equality sign is set in Eq. (1). The typical gravity-gradient value at Earth surface 
is about $3 \times 10^{-6} \mathrm{~s}^{-2}$. The tide contribution of nearby masses must also be carefully considered in relation to the specific experimental setup; here only the compensating masses are assumed to be in the neighborhood of the region of interest.

\section{FLATTENING EARTH ACCELERATION}

To counteract the gravity gradient generated by Earth over an axially extended region, the natural candidate is a ring mass coaxial with the same region, and with local gravity. The gravity gradient of such a mass at the center of the configuration is also a tensor with vanishing off-diagonal elements. As for the spherical Earth contribution, and as a consequence of the solenoidal nature of the gravitational field in mass-free space, the vertical gradient is twice that of the horizontal, but the sign of the tensor is opposite with respect to that of Earth. As noted in [14], the tensor gravity-gradient field at the center of a mass ring is the same as the field that would be produced by a negative mass placed below that point. Reducing the axial gradient $\Gamma_{V}$ by means of a mass ring thus brings the same decrease factor to the radial gradient $\Gamma_{H}$. Because of the symmetry of the problem, a description in the cylindrical coordinate system is adopted where the $z$ axis coincides with the gravity-gradient stabilization direction and the origin is at the center of the compensation region. The discussion is then restricted to the half-plane $\phi=0$.

Once the basic building element of the gravity compensator is defined, the physical and geometrical constraints of the problem must be analyzed. First considerations include the density and the density homogeneity of the mass compensator. A detailed analysis of the material choice is beyond the scope of the present article; as a basic rule it must be taken into account that a high density allows for a compact compensation configuration, and high homogeneity is an advantage.

Geometric constraints include the extension of the axial region to be compensated, and the boundaries of the region that can be occupied by the compensator mass. The allowed region is in general a square torus, defined by an internal and an external radius, and by an axial extension, symmetric with respect to the origin for the sake of simplicity. The internal radius boundary is necessary to house the vacuum tube of the atomic fountain, possible magnetic coils, magnetic shields, and electrodes.

The next step is to find the optimal mass distribution that fulfills the physical and geometrical requirements. Different parameters can be adopted to evaluate the configurations: (1) the maximum acceleration gradient in the region $L$ where a flat acceleration is pursued; (2) the difference between the maximum and the minimum acceleration over $L$; (3) the mean square variance of the acceleration with respect to its average value over $L$. A mixed strategy showing the fastest convergence to the best configuration was chosen: first, the condition of flat average acceleration is obtained (this condition is verified when the acceleration has the same value at the two extremes of $L$ ), and then the residual roughness of the acceleration is smoothed by minimizing the last of the three previous parameters.

The search for the optimal configuration is carried out with a numerical optimization technique, since the problem is too complex to be treated analytically. The square toroidal region suitable for the compensator is parameterized by approximating it with $N$ finite mass rings. Fine radial and axial steps reduce systematic effects given by the mass discretization. The $k$ th ring has a linear mass density $\lambda_{k}$ which satisfies the relation $\lambda_{k} \leqslant \lambda_{\max }\left(\lambda_{\max }\right.$ is given by the maximum density and the adopted ring section), and its position is given by the vector $\mathbf{r}_{k}=\left[\rho_{k}, z_{k}\right]$, which describes the intersection of the ring center with the half-plane $\phi=0$. With the ring coaxial to the gravity-gradient compensation axis, $\rho_{k}$ is its radius and $z_{k}$ is the axial distance of its center from the origin.

Each mass ring produces a vertical acceleration at $z$ on the axis:

$$
g_{\rho_{k}, z_{k}}(z) \equiv 2 \pi \lambda_{k} G \frac{\rho_{k}\left(z_{k}-z\right)}{\left[\rho_{k}^{2}+\left(z_{k}-z\right)^{2}\right]^{3 / 2}} .
$$

The axial gradient of the previous formula gives

$$
\Gamma_{\rho_{k}, z_{k}}(z) \equiv \frac{\partial g_{\rho_{k}, z_{k}}(z)}{\partial z}=2 \pi \lambda_{k} G \rho_{k} \frac{2\left(z_{k}-z\right)^{2}-\rho_{k}^{2}}{\left[\rho_{k}^{2}+\left(z_{k}-z\right)^{2}\right]^{5 / 2}},
$$

and the average gradient the ring produces on the axis in the region $-a<z<a$ is then

$$
\left\langle\Gamma_{\rho_{k}, z_{k}}\right\rangle \equiv \frac{1}{2 a} \int_{-a}^{a} \Gamma_{\rho_{k}, z_{k}}(z) d z=\frac{1}{2 a}\left[g_{\rho_{k}, z_{k}}(a)-g_{\rho_{k}, z_{k}}(-a)\right]
$$

The numerical procedure to optimize the mass distribution is based on the simulated-annealing Metropolis algorithm [16]. The first step consists of filling the region where the mass rings generate a negative average gravity gradient over the region of interest $L$ with the maximum allowed density in each ring. In the example presented here, $L$ is defined by setting $a=5 \mathrm{~cm}$, and the region verifying $\left\langle\Gamma_{\rho_{k}, z_{k}}\right\rangle \leqslant 0$ is that within the two dashed lines in Fig. 2. The filling procedure systematically selects the rings within the allowed region by increasing the radius, so as to pick up bigger contributions to the gradient compensation first. As an example, in Fig. 1 the residual average gradient over $L$ is plotted versus the external radius of the filled region, once the internal radius is set (shown are the cases of radius 4,6 , and $8 \mathrm{~cm}$ ). The filling process stops when the average gradient over $L$ crosses the zero: beyond that Earth's gradient would be overcompensated. The second part in Fig. 1 shows the vertical acceleration obtained once the compensation condition is reached. As the inset points out, the average acceleration over $L$ is flat, and the residual inhomogeneity is already of the order of $5 \%$ of its initial value for the three configurations. Notably, the homogeneity improves for bigger internal radius, at the expense of a bigger size and mass.

If the allowed region with the correct sign contribution to the gravity gradient is completely filled before the zero-order compensation is reached, the procedure stops. Otherwise, it is the starting point of a Monte Carlo optimization algorithm. The evolution is carried out in this way: at each step, two rings are randomly chosen within the allowed region; the two relative average gradients over $L$ are calculated; the two rings' mass densities are scaled so as to provide a vanishing contribution to the average gradient when summed together. The two density values are scaled with respect to a preset value $d \lambda$, and their global sign is chosen so as to decrease 

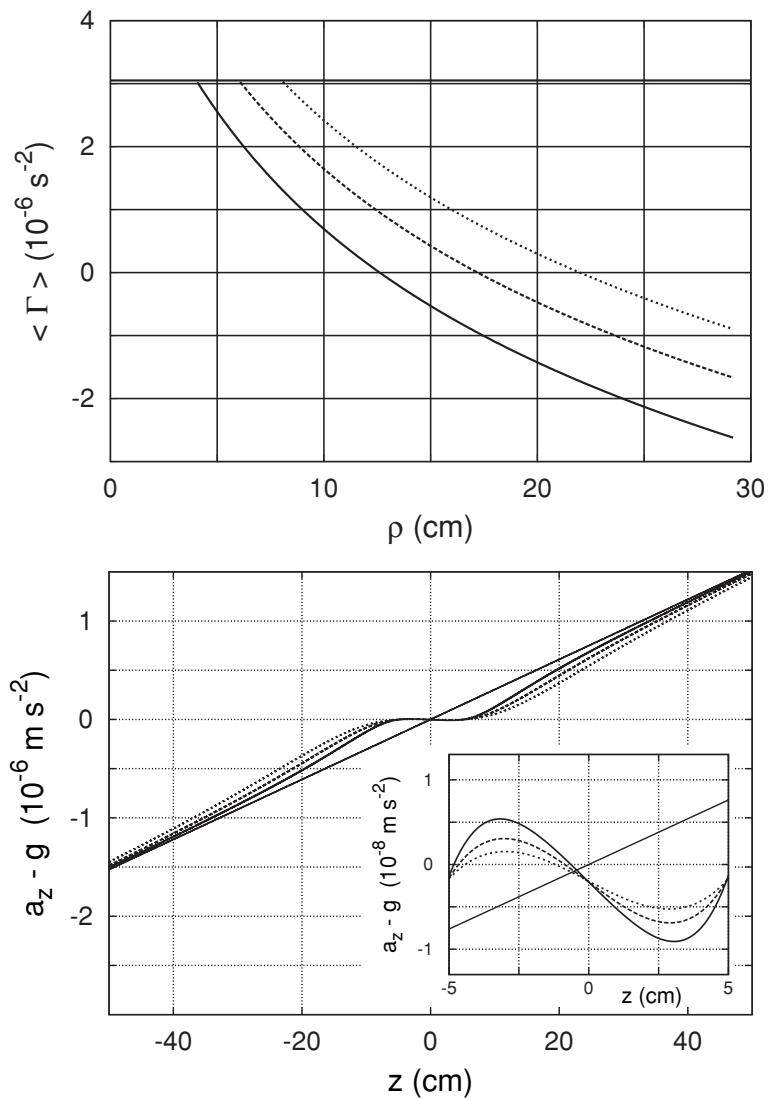

FIG. 1. (Top) Average acceleration gradient over the region of interest (between -5 and $5 \mathrm{~cm}$ ) as a function of the external radius of the compensator, when only the region with negative gradient is filled with mass. Shown are the cases of internal radius fixed to 4 (solid), 6 (dashed), and $8 \mathrm{~cm}$ (dotted). The horizontal solid line is at Earth gravity-gradient value. (Bottom) Residual acceleration on the axis for the three previous configurations, with the external radius fixed by the passage through the zero of the upper curves. The thin solid straight line represents Earth acceleration. The inset shows the acceleration in the region of interest; here the slope of Earth acceleration is divided by 20 for comparison.

the mean square variance of the gravity gradient over $L$. The couple of densities is then added to the density matrix, with a further normalization if the condition $0 \leqslant \lambda_{k} \leqslant \lambda_{\max }$ is not verified. The evolutionary step is then repeated. The parameter $d \lambda$ is progressively lowered along the evolution, following a simulated annealing schedule defined on a trial-and-error approach to optimize the convergence to the optimal solution.

Figure 2 shows an example of the best mass compensator obtained for different external radii. The conditions adopted for the calculation are density $10^{4} \mathrm{~kg} / \mathrm{m}^{3}$, internal radius $R_{\text {int }}=$ $4 \mathrm{~cm}$, half-height of the allowed region for the compensator $13 \mathrm{~cm}$, and half-height of the compensation region $a=5 \mathrm{~cm}$. The section of each mass ring is $0.1 \times 0.1 \mathrm{~mm}^{2}$, whereas the resulting acceleration is calculated over a grid with step $0.1 \mathrm{~mm}$. The first plot shows the configuration reached at the conclusion of the initial filling procedure, which occurs for $R_{\text {ext }}=12.6 \mathrm{~cm}$. In the next two plots, the external radius is set to 13.5 and $14.2 \mathrm{~cm}$. Notably, the optimization procedure always makes use of the larger radius mass rings verifying $\left\langle\Gamma_{\rho_{k}, z_{k}}\right\rangle \leqslant 0$, since their contribution over $L$ is more
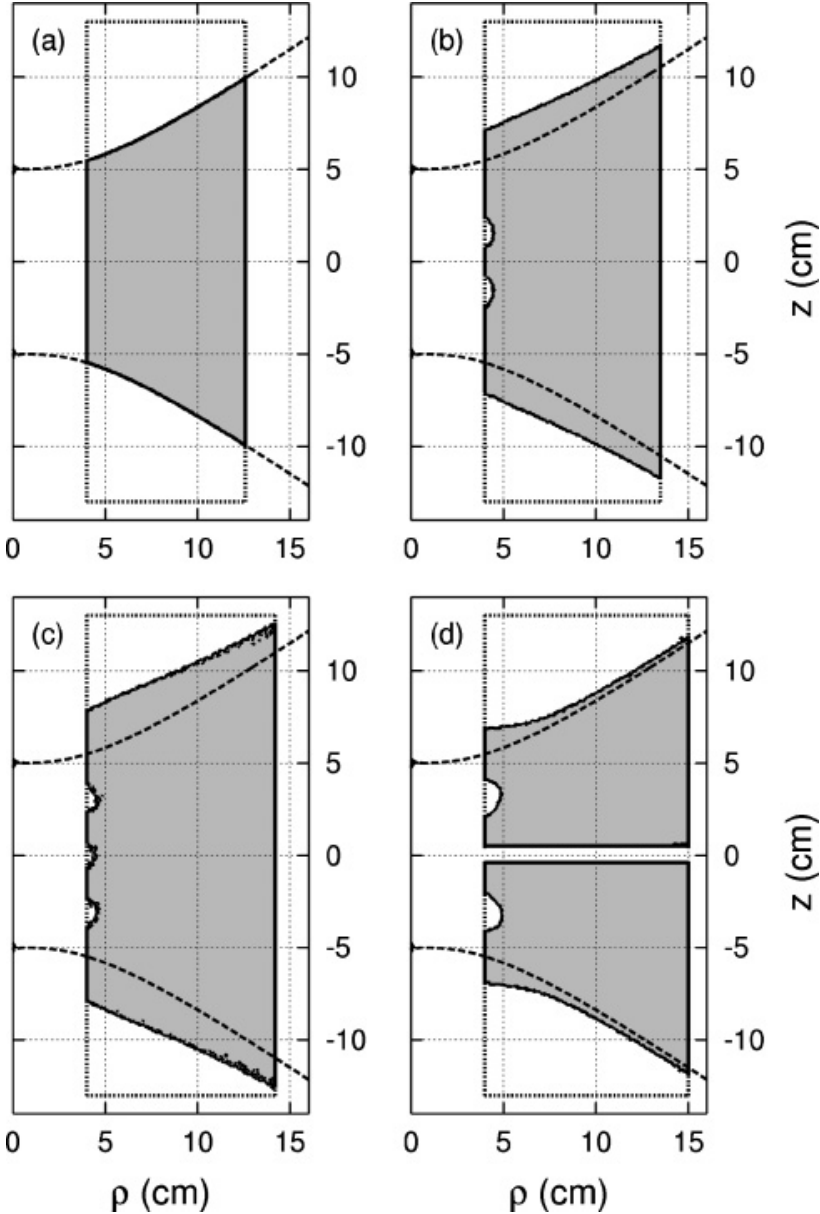

FIG. 2. The two dashed lines represent the set of mass rings giving vanishing average gradient $\left\langle\Gamma_{\rho_{k}, z_{k}}\right\rangle$ on the region of interest $L$, as given by Eq. (5) when $a=5 \mathrm{~cm}$. The dotted lines are the geometric constraints on the compensation mass. The internal radius of the configurations is set to $4 \mathrm{~cm}$, the axial borders are at $\pm 13 \mathrm{~cm}$, the external radius is set to $12.6,13.5,14.2$, and $15 \mathrm{~cm}$ from (a) to (d). In the last image, there is an aperture of $1 \mathrm{~cm}$ on the horizontal symmetry plane of the configuration. The gray region is filled with mass, as determined by the optimization algorithm. The total mass of each configuration is $70,101,117$, and $116 \mathrm{~kg}$ from (a) to (d).

homogeneous. The gravity gradient overcompensation these rings cause is canceled in two different ways: by adding mass rings outside of the dashed boundaries, and by removing the highly inhomogeneous contributions of close components. The underlying random selection process works to smooth the residual acceleration curve. The resulting acceleration in the compensation region for the configurations is presented in Fig. 3, where Earth's acceleration with a gradient reduced by a factor 100 is plotted for comparison. Considering the region between -4 and $4 \mathrm{~cm}$, noteworthy reductions in residual gravity gradient by factors of about $10^{2}$ and $10^{3}$ are achieved for the configuration having external radius 13.5 and $14.2 \mathrm{~cm}$, respectively. The acceleration can be further smoothed by running the optimization after increasing the allowed region for the mass compensator.

The optimization algorithm can handle more complex geometrical constraints by defining a suitable multiplicative loss function for the evaluation of the configuration efficiency. 


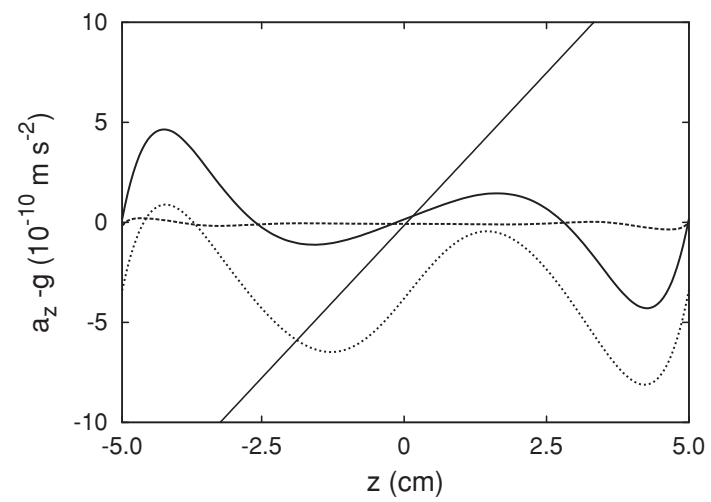

FIG. 3. Residual acceleration on the axis for the configurations (b), (c), and (d) in Fig. 2, respectively, in solid, dashed, and dotted line. The thin straight line with positive slope is shown for reference and represents Earth's acceleration, when its gravity gradient is divided by a factor 100 .

Such function is unitary within the allowed space region, whereas in the forbidden regions it increases the negative contributions to the efficiency and decreases the positive ones. An exponential growth for the suppression effect over a suitable length scale can be defined as in [17], where the shape of a magnetic trap for neutral atoms is optimized. As an example, in Fig. 2(d) a 1-cm-high optical access is imposed on the horizontal symmetry plane of the compensation mass. The resulting compensation factor for the gravity gradient is a remarkable $10^{2}$.

The result of the optimization algorithm are configurations where the mass is confined in well-defined regions having the maximum allowed density. The precise realization of the compensator masses by machining is therefore feasible.

\section{ATOM INTERFEROMETERS}

In fountain-based atom interferometry the fluctuations of the initial position and velocity of the atomic clouds cause a phase noise that eventually limits the measurement sensitivity. Considering a $\pi / 2-\pi-\pi / 2$ sequence with a time interval $T$ between the pulses, the phase shift given by an initial vertical displacement $\delta z$ is $-k_{\mathrm{eff}} T^{2} \Gamma_{V} \delta z$, where $\hbar k_{\mathrm{eff}}$ is the momentum difference between the wave-function trajectories, and $\Gamma_{V}$ the vertical component of the gravity gradient as defined in Eqs. (2). The phase contribution due to an initial velocity shift $\delta v_{z}$ is $-k_{\mathrm{eff}} T^{3} \Gamma_{V} \delta v_{z}$. In [10] a 10-m atomic gravity gradiometer to test atom and neutron neutrality is proposed, and Earth's gravity gradient is assumed to be reduced to $10 \%$ over the whole measurement region by an engineered local mass distribution. Even so, the atomic position and velocity must be controlled at the $1-\mu \mathrm{m}$ and $1-\mu \mathrm{m} / \mathrm{s}$ levels, respectively, for shot-noise-limited sensitivity. Moreover, if the sensitivity is improved toward the Heisenberg limit by employing entangled atomic states [18], the control degree of the atomic dynamics must scale proportionately.

By flattening Earth's acceleration over $10 \mathrm{~cm}$, the mass configurations of the previous section strongly reduce the dynamics-induced noise for interferometers with a pulse spacing of the order of $100 \mathrm{~ms}$ symmetric with respect to the $\pi$ pulse, since these are completely realized within the flattened region. However, the sensitivity of an atom interferometer scales with the square of the pulse spacing, therefore, increasing the free-fall interval is a straightforward way to obtain better precision. The solution presented so far to reduce the phase noise determined by the gravity gradient soon becomes impracticable, because of the total mass required. For a 1-m compensation and a mass-free internal radius of $4 \mathrm{~cm}$, the mass of the zero-order compensator is about $10^{4} \mathrm{~kg}$, and the residual gravity gradient is $30 \%$ with respect to Earth's.

The fact that the gravity gradient couples the atomic position and velocity to the interferometric phase only when the pulses are delivered is exploited to solve this problem. For a three-pulse interferometer symmetric with respect to the apogee, it is then possible to devise a configuration with only two $10-\mathrm{cm}$ compensators, one in the axial region where the two $\pi / 2$ pulses interact with the cloud, and one at the top of the trajectory where the $\pi$ pulse acts. In this way, an effective compensation of the gravity gradient at levels comparable with those shown in the previous section is obtained also for very tall configurations. Notably, the relative positioning of the two compensators (which can be done at the $\mu \mathrm{m}$ level by adopting stiff supports and optical interferometry measurements) affects the accuracy of the phase measurement, but not the precision. The required control degree for the atomic position and velocity is then strongly relaxed.

This multimass compensation scheme can be adapted to the case characterized by a large spatial splitting at the time of the $\pi$ pulse, as shown in Fig. 4. In the example presented, the interrogation time of the experiment is $T=1.16 \mathrm{~s}$, a velocity splitting of $\hbar k_{\text {eff }} / m_{\text {atom }} \sim 1 \mathrm{~m} / \mathrm{s}$ is adopted (allowed by improved large momentum transfer beam splitters [19]), and this results in an $\sim 1-\mathrm{m}$ splitting at the apogee. The pulse timing is chosen to send the $\pi$ pulse not at the top of the cloud trajectories to avoid degeneracy between oppositely directed $k_{\text {eff }}$ in the case of retroreflected Raman beams. Four mass compensators are required, one for each region where the atomic wave function is manipulated by the laser field. The $1-\mathrm{m} / \mathrm{s}$ velocity splitting requires about 85 pulses in

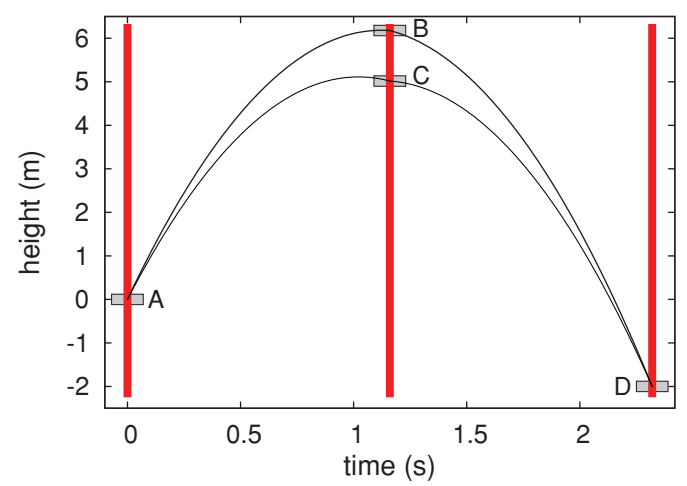

FIG. 4. (Color online) Atom wave-function trajectories versus time for a gravimeter implementing a $\pi / 2-\pi-\pi / 2$ pulse sequence. The pulse spacing is $T=1.16 \mathrm{~s}$, the velocity splitting between the two paths is $\delta v=1 \mathrm{~m} / \mathrm{s}$, and the vertical velocity at the time of the first $\pi / 2$ pulse is $11 \mathrm{~m} / \mathrm{s}$. In gray, and labeled (A)-(D), are shown the four regions on the axis where atoms interact with Raman pulses and where compensator masses are placed to locally flatten Earth's acceleration. 
the case of rubidium atoms and Raman lasers close to the $D_{2}$ line. Given a $10-\mathrm{cm}$ vertical region for each composite pulse, and with the maximum atomic velocity during the interferometer sequence of $11 \mathrm{~m} / \mathrm{s}$, the time interval for each optical pulse is $100 \mu \mathrm{s}$. Assuming a compensation of Earth's gravity gradient to $0.1 \%$ in each $10-\mathrm{cm}$ region, the initial position fluctuations of the atomic cloud have to be controlled at the $100-\mu \mathrm{m}$ level and the initial velocity at the $100-\mu \mathrm{m} / \mathrm{s}$ level for shot-noise-limited sensitivity. These values must scale accordingly to the sensitivity improvement given by the use of entangled sources. Additional masses must be placed in the case of a gravity gradiometer employing two clouds flying at a given distance and subjected to the same laser pulse sequence.

\section{CONCLUSIONS}

A method to lower Earth's tidal effect over extended axial regions has been presented that achieves reductions in the residual gradient over $10 \mathrm{~cm}$ by factors of $10^{3}$ and more. In the case of fountain atom interferometry, a smooth acceleration region allows the reduction of the statistical effect of atom positioning: the interferometric phase contains two terms coupling the gravity gradient to the atomic initial position and initial velocity. The method is further adapted to cope with tall Raman atom interferometers, and more specifically for the cases in which flattening the acceleration over the entire atomic trajectory would require a prohibitively heavy mass. The approach relies on a multimass scheme that compensates the gravity gradient only in the regions where atoms interact with the interferometric pulses. By strongly relaxing the requirements on the atomic dynamics side, the compensation method can play an important role in obtaining the very high sensitivities targeted in several atom interferometry experiments being developed nowadays.

\section{ACKNOWLEDGMENTS}

The author thanks G. B. Partridge for critical reading of the manuscript. This work was supported by the European Union (IEF Grant No. PIEF-GA-2009-235375) and by IFRAF.
[1] G. Santarelli, P. Laurent, P. Lemonde, A. Clairon, A. G. Mann, S. Chang, A. N. Luiten, and C. Salomon, Phys. Rev. Lett. 82, 4619 (1999).

[2] A. Peters and S. Chu, Nature 400, 849 (1999).

[3] J. M. McGuirk, G. T. Foster, J. B. Fixler, M. J. Snadden, and M. A. Kasevich, Phys. Rev. A 65, 033608 (2002).

[4] S. Fray, C. A. Diez, T. W. Hänsch, and M. Weitz, Phys. Rev. Lett. 93, 240404 (2004).

[5] H. Müller, A. Peters, and S. Chu, Nature 463, 926 (2010).

[6] J. B. Fixler, G. T. Foster, J. M. McGuirk, and M. A. Kasevich, Science 315, 74 (2007).

[7] G. Lamporesi, A. Bertoldi, L. Cacciapuoti, M. Prevedelli, and G. M. Tino, Phys. Rev. Lett. 100, 050801 (2008).

[8] H. Marion et al., Phys. Rev. Lett. 90, 150801 (2003).

[9] S. Dimopoulos, P. W. Graham, J. M. Hogan, and M. A. Kasevich, Phys. Rev. Lett. 98, 111102 (2007).

[10] A. Arvanitaki, S. Dimopoulos, A. A. Geraci, J. Hogan, and M. Kasevich, Phys. Rev. Lett. 100, 120407 (2008).
[11] S. Dimopoulos, P. W. Graham, J. M. Hogan, M. A. Kasevich, and S. Rajendran, Phys. Lett. B 678, 37 (2009).

[12] A. Bertoldi, G. Lamporesi, L. Cacciapuoti, M. de Angelis, M. Fattori, T. Petelski, A. Peters, M. Prevedelli, J. Stuhler, and G. M. Tino, Eur. Phys. J. D 40, 271 (2006).

[13] C. W. Stubbs, E. G. Adelberger, B. R. Heckel, W. F. Rogers, H. E. Swanson, R. Watanabe, J. H. Gundlach, and F. J. Raab, Phys. Rev. Lett. 62, 609 (1989).

[14] R. L. Forward, Phys. Rev. D 26, 735 (1982).

[15] R. Friedberg, Phys. Rev. D 36, 386 (1987).

[16] N. Metropolis, A. Rosenbluth, M. Rosenbluth, A. Teller, and E. Teller, J. Chem. Phys. 21, 1087 (1953).

[17] L. Ricci, A. Bertoldi, and D. Bassi, Rev. Sci. Instrum. 73, 3181 (2002).

[18] D. Leibfried, M. D. Barrett, T. Schaetz, J. Britton, J. Chiaverini, W. M. Itano, J. D. Jost, C. Langer, and D. J. Wineland, Science 304, 1476 (2004).

[19] J. M. McGuirk, M. J. Snadden, and M. A. Kasevich, Phys. Rev. Lett. 85, 4498 (2000). 